\title{
Line mixing and collision induced absorption in the oxygen A-band using cavity ring-down spectroscopy
}

\author{
Frans R. Spiering, ${ }_{1}^{1, a)}$ Maria B. Kiseleva, ${ }^{2}$ Nikolay N. Filippov, ${ }^{2}$ Hans Naus, ${ }^{3}$ \\ Bas van Lieshout, ${ }^{1}$ Chris Weijenborg, ${ }^{1}$ and Wim J. van der Zande ${ }^{1}$ \\ ${ }_{1}^{1}$ Institute for Molecules and Matter, Radboud University, Nijmegen 6525AJ, The Netherlands \\ ${ }^{2}$ St. Petersburg State University, 199034 St. Petersburg, Russia \\ ${ }^{3}$ OGS Systems, The Netherlands
}

(Received 16 November 2009; accepted 16 June 2010; published online 21 September 2010)

\begin{abstract}
This paper reports on the absorption of molecular oxygen in the region of the A-band near $760 \mathrm{~nm}$ under atmospheric conditions relevant for satellite retrieval studies. We use pulsed laser cavity ring-down spectroscopy with a narrow bandwidth laser and use pressure scans to increase the accuracy of the measured oxygen extinction coefficients. Absolute binary absorption coefficients in minima between absorption lines of the A-band spectrum have been measured and tabulated. We use the so-called adjustable branch coupling model including line mixing to calculate the magnetic dipole absorption in order to determine the contribution of collision induced absorption. The line mixing model has been optimized such that the collision induced absorption spectrum is smooth.

(C) 2010 American Institute of Physics. [doi:10.1063/1.3460924]
\end{abstract}

\section{INTRODUCTION}

Absorption of radiation by molecular oxygen has been the subject of numerous studies in the past. Oxygen is an unusual molecule having a triplet ground state besides five other low lying excited states. None of these six low lying states are coupled by allowed electric dipole transitions. Despite the forbidden nature of these transitions, the amount of oxygen in the atmosphere is so high that oxygen absorbs solar light in our atmosphere efficiently. This absorption by oxygen, in particular in the near-infrared part of the spectrum (the oxygen A-band) has become important in satellite retrieval studies. ${ }^{1}$ It is expected that the next generation of atmospheric satellite missions must be able to characterize the composition of the troposphere with such an accuracy that local emissions of carbon dioxide $\left(\mathrm{CO}_{2}\right)$ can be determined from observed column densities. Determination of the effective oxygen column is required in order to obtain an accurate air mass factor, which corrects for the geometry of satellite and the sun and for the effects of aerosol scattering, clouds and surface albedo. Accurate $\mathrm{CO}_{2}$ columns $(<1 \%)$ require very accurate $(<0.2 \%)$ oxygen columns and the latter implies the need for high accuracy data on oxygen absorption including collision effects. ${ }^{2}$ Satellite instruments, which record the A-band spectrum often at low resolution, detect column density differences from variations of the transmittance in the weak minima in between the sharp resonances. Here, the absorption coefficient ranges from $10^{-8}$ to $10^{-5} \mathrm{~cm}^{-1}$.

Molecular collisions give rise to different effects that influence the absorption coefficient and the shapes of the sharp absorption lines. The best known effect is pressure broadening in the form of a Lorentzian line parameter. Thermal motion adds a Gaussian contribution to the line shape.

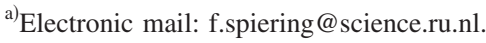

Next to these well known effects on the line shape, more subtle effects are present, such as Dicke narrowing, ${ }^{3}$ line mixing, (LM) ${ }^{4}$ and collision induced absorption (CIA). ${ }^{5}$ The first two effects only redistribute the absorption coefficient as function of wavelength thereby changing the line shapes. CIA describes the absorption by transient $\mathrm{O}_{2}-\mathrm{O}_{2}$ collision complexes. The short duration of the collision and the exchange of collision energy and internal energy result in a weak absorption spectrum without sharp features. Being the direct result of binary collisions, CIA increases strictly quadratic with density.

Most earlier studies on oxygen A-band absorption focused on spectroscopic parameters of individual rotational lines from high resolution spectra obtained by means of Fourier transform or laser spectroscopy (see, for example, Brown et al. ${ }^{6}$ Robichaud et al. ${ }^{7}$ Predoi-Cross et al. ${ }^{8}$ and references therein). Predoi-Cross et al. ${ }^{8}$ analyzed the A band line shapes using a high resolution FT technique. In the analysis of their results, they used various line shapes (Galatry, ${ }^{9}$ Rautian Sobel'mann ${ }^{10}$ and speed dependent Rautian Sobel'mann) which take into account the Dicke effect and velocity dependent effects. However, in order to fit their lines within the experimental noise level they still needed to add line mixing.

The number of studies concentrating on collision induced effects is small. In fact, until relatively recently, the presence of an observable CIA contribution was even questioned. Tabisz et al. ${ }^{11}$ were the first to report a CIA contribution deduced from measurements at high densities [100-300 amagat ${ }^{12}$. Later, Greenblatt et al. ${ }^{13}$ reported CIA to be negligible. In a recent work, Tran et al. ${ }^{14}$ quantified the CIA spectrum. They used a $0.5 \mathrm{~cm}^{-1}$ resolution Fourier Transform spectrometer and oxygen densities up to 150 amagat. To the best of our knowledge, Tran et al. were the first to use a quantitative LM model to extract the CIA contribution from the observed absorption in the region of the A-band.

In this paper, we report on absolute measurements in 


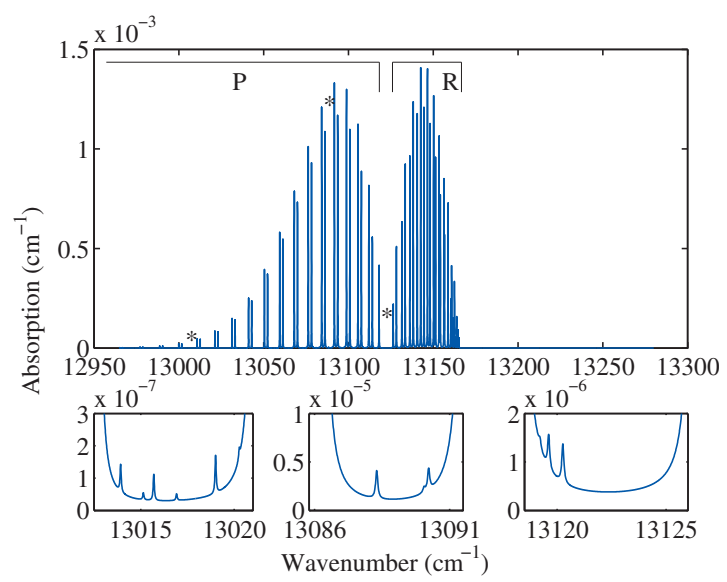

FIG. 1. Spectrum of the A-band at 1 amagat calculated using the ABC model. The magnitude and shape of the absorption coefficients in two minima between $\mathrm{P}$ lines and in the region of the missing Q-branch are illustrated. The selected minima are indicated by the asterisk in the A-band spectrum

between the P-lines of the A-band and in the region beyond the bandhead $\left(13165 \mathrm{~cm}^{-1}\right)$ as well as in the gap of the missing Q-branch of the pressure dependent absorption coefficients using pulsed laser cavity-ring down spectroscopy. Oxygen pressure ramps starting at a few millibars and ending between 1 and 5 bar were used. Our measurements make it possible to reconstruct the CIA spectrum below the oxygen A-band. We argue that the introduction of LM in the calculations of line shapes in the A-band is necessary and we use a model called the adjustable branch coupling (ABC) model to introduce LM. This model has been developed by Tonkov and co-workers. ${ }^{15,16}$

\section{THEORY}

The oxygen A-band (13 000-13 $\left.200 \mathrm{~cm}^{-1}\right)$ stems from the $b^{1} \Sigma_{g}^{+}(v=0) \leftarrow X^{3} \Sigma_{g}^{-}(v=0)$ transition. It is a weak magnetic dipole transition, strongly electric dipole forbidden. Babcock and Herzberg identified the origin of the A-band transition correctly in $1949 .{ }^{17}$ The spectrum contains a P-branch and an R-branch, related to changes in the rotational angular momentum $N, \Delta N=N^{\prime \prime}-N^{\prime}= \pm 1$. A Q-branch is missing. The triplet nature of the electronic ground state results in a doubling of the P-lines into PP and PQ transitions and of the R-lines in RR and RQ transitions, related to the $J=N+S$, allowing for $\Delta J=J^{\prime \prime}-J^{\prime}=0, \pm 1$, for fixed $\Delta N$. The magnitude of the splitting is $\sim 2 \mathrm{~cm}^{-1}$. Finally, as is well known for molecular oxygen only odd $N$ levels exist in the ground state. The excited $b$-state only has even $N$ values. Figure 1 contains the A-band spectrum at 1 amagat. The three small figures show examples of the spectrum that have been studied, namely in between the P-lines and at the position of the missing Q-branch. The different naturally occurring oxygen isotopologues are clearly visible and need to be taken into account in the analysis.

\section{A. Collisional effects}

Absorption lines are modified by collisions between oxygen molecules, and thus by the oxygen-oxygen interac- tion potential, of which the details determine the rate of velocity changing and of rotationally inelastic collisions. In approximate models, the effects of these collisions are accumulated under the name of collision broadening and parametrized in a single parameter: the collisional line width. The resulting Lorentzian lines are convoluted with the Gaussian shaped Doppler contribution into a Voigt line profile. Dicke ${ }^{3}$ and others have shown that the Doppler broadening can be pressure dependent. Rautian has explained this phenomenon in terms of diffusion on a quantum mechanical scale, analogous to the interpretation of the Mossbauer effect. ${ }^{18}$ Galatry ${ }^{9}$ as well as Rautian and Sobel'man ${ }^{10}$ came up with schemes to include Dicke narrowing into the Voigt profile quantitatively. All these corrections give dominating effects near the line center, whereas the line wings are generally much less affected. Only LM has a dominating relative effect on the absorption coefficients in the line wings. As we accurately determine the absorption coefficients at the line wings, we concentrate on introducing LM in order to determine the local contribution of the CIA. LM takes into account the effect of inelastic collisions on the line shape, which results in intensity transfer between rotational lines. Fano was the first to derive an expression for the effect of LM on Lorentz lines. ${ }^{4}$ He derived the following expression for the spectral distribution:

$\Phi(\omega)=\frac{1}{\pi} \mathfrak{R e}\left\{\sum_{q j, q^{\prime} j^{\prime}} \rho_{j} x_{q j^{\prime}} x_{q^{\prime} j^{\prime}}\left[\frac{1}{i\left(\omega-L_{0}\right)+\Gamma(\omega)}\right]_{q j, q^{\prime} j^{\prime}}\right\}$.

In this equation, $\rho_{j}$ are the initial state populations, related to the temperature, $x_{q j}$ are the so-called reduced dipole moments. The products $\rho_{j}\left|x_{q j}\right|^{2}$ provide the line strengths as found in databases such as HITRAN. ${ }^{19,20} \omega$ is the frequency considered. The labels $q$ and $q^{\prime}$ are used to distinguish transitions within the two individual branches, the remaining label $j$ enumerates all optical transitions. $L_{0}$ is also known as the Liouville operator, which in matrix representation is diagonal containing all transition frequencies. The matrix $\Gamma$ is the relaxation matrix, which contains the effect of collisions on the line shapes. The diagonal of the relaxation matrix contains pressure shifts and collisional broadening coefficients and the off-axis elements contain line mixing effects. We note that a diagonal relaxation matrix ignores line mixing, such that Eq. (1) describes a spectrum consisting of a sum of Lorentzian lines. Assuming hard collisions, the relaxation matrix can be approximated by ${ }^{21}$

$$
\Gamma_{q q^{\prime} j j^{\prime}}=\bar{\tau}^{-1}\left(\delta_{q q^{\prime}} \delta_{j j^{\prime}}-A^{-1} \rho_{j}^{\prime} x_{q j^{j}} x_{q^{\prime} j^{\prime}}\right) .
$$

On the assumption that there is no coupling between the lines belonging to different branches, the relaxation matrix takes the form

$$
\Gamma_{q q^{\prime} j j^{\prime}}=\delta_{q q^{\prime}} \bar{\tau}^{-1}\left(\delta_{j j^{\prime}}-A_{q}^{-1} \rho_{j}^{\prime} x_{q j^{j}} x_{q^{\prime} j^{\prime}}\right) .
$$

The $\delta$ function in $q, q^{\prime}$ reflects that in this form no line mixing takes place between $\mathrm{P}$ - and R-lines. In these equations $\bar{\tau}^{-1}$ is the averaged collision frequency, which can be obtained from the pressure broadening parameters in databases such as HITRAN. $A$ is the total integrated intensity of the band 
and $A_{q}$ is the integrated intensity of branch $q$. It is noted that all components of the relaxation matrix may be complex, providing both lineshifts and line shape changes. An often used simplification is to ignore the imaginary part of the diagonal elements, the pressure induced line shifts, for calculation of the off-diagonal components. Tonkov and co-workers ${ }^{15}$ introduced a refinement of the above expression by adding one parameter, $r$, to allow for the line mixing between transitions between the two branches

$$
\begin{aligned}
\Gamma_{q q^{\prime} j j^{\prime}}= & (1-r) \delta_{q q^{\prime}} \bar{\tau}^{-1}\left(\delta_{j j^{\prime}}-A_{q}^{-1} \rho_{j}^{\prime} x_{q j} x_{q^{\prime} j^{\prime}}\right) \\
& +r \bar{\tau}^{-1}\left(\delta_{q q^{\prime}} \delta_{j j^{\prime}}-A^{-1} \rho_{j}^{\prime} x_{q j^{\prime}} x_{q^{\prime} j^{\prime}}\right),
\end{aligned}
$$

This model has been successfully applied to the $60 \mathrm{GHz}$ band of oxygen, and infrared transitions in $\mathrm{CO}, \mathrm{CO}_{2}, \mathrm{CH}_{3} \mathrm{~F}$, and $\mathrm{CH}_{4}$ (Refs. 15, 16, and 21-23) and is known as the adjustable branch coupling model (or ABC-model). In the case of the oxygen A-band, especially the shape of the spectrum at the missing Q-branch region is strongly affected by the branch coupling coefficient. As we will show, this region is important in assessing the optimal value for $r$ in order to generate a smooth CIA spectrum. The coupling parameter forms the only free parameter used, all the others came from HITRAN.

The last step in the procedure to generate a full spectrum is to take into account the Doppler contribution to the spectrum. Our line mixing model averages over all collisions. We use a Voigt convolution between the line mixing spectrum and the Gaussian contribution associated with the Doppler effect. In practice, our computational procedure determines the line mixing correction on the sum of Voigt line shapes spectrum based on the collisional parameters, including pressure shifts, for each line obtained from the HITRAN. The correction itself results from diagonalization of the line mixing matrix, which is equivalent to a Lorentzian line.

All the above pertains only to the effect of collisions on the line shapes of the (magnetic dipole) allowed A-band transitions. The integrated cross section is unchanged and remains linear in the oxygen density. However, collisions also lower the symmetry of the individual oxygen molecules and deform the electron wave functions slightly. Tipping and co-workers ${ }^{24,25}$ have estimated the effect of the quadrupole interaction induced dipole mechanism. Whereas this mechanism describes the effect of other perturbers such as $\mathrm{N}_{2}$ and $\mathrm{CO}_{2}$ quite well, the authors acknowledge that the self-effect of $\mathrm{O}_{2}-\mathrm{O}_{2}$ collisions is not predicted very well. In all models a CIA spectrum is expected to be without rotational structure as a consequence of the very short duration of the collisions. This collision induced part of the oxygen spectrum is one of the important outcomes of the research presented here.

\section{EXPERIMENT}

\section{A. Experimental method}

To measure oxygen absorption in the region of the A-band we used a cavity ring-down (CRD) spectroscopy technique. Introduced in 1988 by O'Keefe and Deacon, ${ }^{26,27}$ this method has been developed into a very sensitive technique for direct measurements of the quantitative character- istics of molecular absorption, using laser sources. CRD methods are based on measurements of the time dependence of intensity of the laser pulse leaking out of a cavity. The decay rate of the signal contains all the information about loss processes from the passive optical cavity. If a monochromatic light pulse is coupled into the cavity, it leaks out of the cavity, in principle, exponentially with time

$$
I(t)=I_{0}(\nu) e^{-t / \tau(\nu)}
$$

where $I_{0}(\nu)$ is the initial intensity of light leaking out and $\tau(\nu)$ is the cavity ring-down time. The value of $\tau(\nu)$ completely depends on the cavity losses

$$
\tau(\nu)=\frac{d}{c} \cdot \frac{1}{|\ln (R)|+\kappa(\nu) l},
$$

where $d$ is the cavity length, $c$ is the speed of light, $R$ is the reflectivity of the mirrors, $\kappa(\nu)$ is the extinction coefficient of the oxygen inside the cavity, and $l$ is the single pass path length (if the cavity is completely filled with oxygen, $l$ is equal to $d$ ). Therefore, the extinction coefficient of oxygen can be obtained as follows:

$$
\kappa(\nu)=\frac{1}{\tau(\nu) c}-\frac{|\ln (R)|}{d},
$$

where the background level $|\ln (R)| / d$ is determined by the losses in the empty cell.

The CRD spectroscopy technique has two remarkable advantages. First, the value of extinction coefficient is determined by the decay rate of the light pulse intensity leaking out of the cavity and is thus independent of the fluctuations of the laser intensity. Second, the use of high reflectivity mirrors gives the cavity an optical path length of several tens of kilometers, while working with a half-meter long cavity. The combination of these advantages results in the very high sensitivity (up to $10^{-9} \mathrm{~cm}^{-1}$ ) of the CRD method. It is important to use a laser with a line width which is narrow with respect to the width of spectral features of the molecule, such that the decay of light from the cavity can be correctly represented by a single exponential function.

\section{B. Experimental setup}

The pulsed cavity ring-down setup ${ }^{26,28}$ used in this work is presented in Fig. 2. We pumped a dye laser (ScanMate from Lambda Physik, Ft. Lauderdale, FL) at $532 \mathrm{~nm}$ with a Nd:YAG (yttrium aluminum garnet) laser operating at $30 \mathrm{~Hz}$ and using pyridine-2. The bandwidth of the laser was typically $0.07 \mathrm{~cm}^{-1}$, without using an etalon. The resulting laser light was guided into a cavity constructed out of two highly reflective mirrors (Research Electro Optics, Boulder, CO), reflectivity $99.999 \%$ at $775 \mathrm{~nm}$, focal length $6 \mathrm{~m}$, placed $\sim 30 \mathrm{~cm}$ apart. In order to study the pressure dependent behavior of the absorption we placed the full cavity including the mirrors inside a home built pressure cell, ensuring that the alignment is not affected by the pressure ramps.

A special system for accurate alignment of the mirrors inside the cell based on linear motors was designed. A flowmeter/controller pair (High Tech: El-Flow) was used to increase the pressure in the cell which was equipped with a 


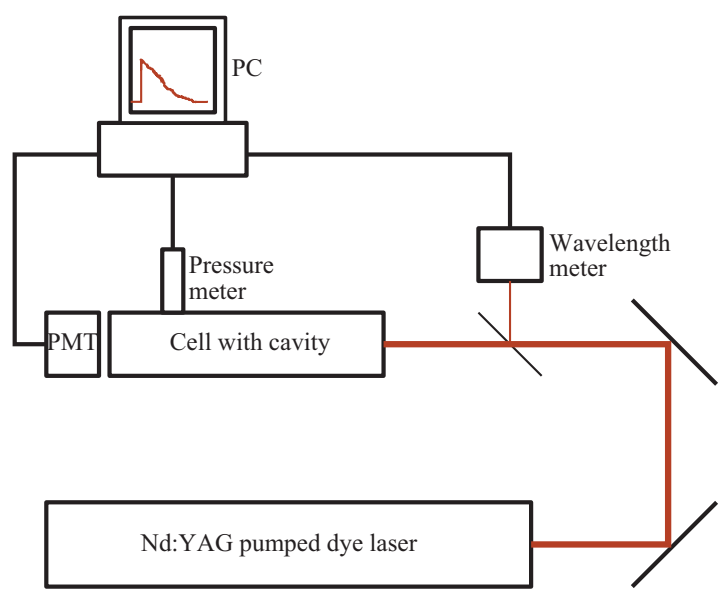

FIG. 2. The experimental setup as used for the determination of density dependent extinction. A ND:YAG laser pumped dye laser is sent into the ring down cavity. Part of the signal is used for wavelength calibration. The ring down signal can be observed with a CCD camera to determine the quality of the signal. Finally, the photomultiplier tube captures the signal. For each shot, wavelength, pressure and PMT signal are stored for analysis.

membrane pump (Edwards XDS5, Tewksbury, MA). The pressure of oxygen $(99,998 \%$ pure) inside the cell was measured with a pressure meter (Pfeifer: Dual Gauge, Nashua, $\mathrm{NH})$ with a relative error of $0.15 \%$ of the reading. The intensity of light leaking out of the CRD cell was detected with a photomultiplier tube (Licel High Dynamic Range Photomultiplier); an appropriate bandpass filter was placed in front of the tube to prevent the detection of surrounding light that could pass through the cell. Part of the light coming out of the CRD cell was also sent on a sensitive charged-coupled device (CCD) camera to control the quality of the alignment of the cavity. This was required for obtaining reproducible results. The data from the photomultiplier tube were transferred to a computer for on-line fitting of the intensity decays and storage of the traces by use of a scope card (GageScope CS14100). Other relevant data, such as the pressure of the sample and the wavelength were recorded simultaneously. The wavelength was measured with a wavelength meter (HighFinesse: Wavelength Meter Ångstrom WS/7) which was calibrated with a single mode temperature stabilized HeNe laser (absolute calibration $0.005 \mathrm{~cm}^{-1}$ ).

\section{Experimental procedure}

To obtain the value of the extinction coefficient of oxygen at a given wavelength within the region of the A-band the following experimental procedure was used: the empty cell was slowly filled with oxygen up to a certain maximum pressure, estimated in advance for each wavelength. The value of the maximum pressure was chosen to ensure a considerable change of the extinction during the pressure scan. During the filling of the cell the pressure of oxygen and the cavity ring-down time were simultaneously recorded. The resulting experimental curves display the density dependence of the extinction of oxygen at a given frequency. The advantage of this procedure is that the loss of the empty cavity is measured during each experiment, resulting in an increased accuracy of the measured extinction coefficient. The experi- mental procedure described above was introduced for measurements of the weak Rayleigh scattering cross sections of nitrogen and sulfur hexafluoride. ${ }^{29}$

Measurements of the extinction coefficient of oxygen were performed within the spectral region from 12960 to $13280 \mathrm{~cm}^{-1}$ in all minima between the rotational lines of the P-branch, in the region between the P- and R-branches and above the bandhead of the R-branch of the A-band of oxygen. The presence of different oxygen isotopologues and their accurate positions were important for a correct retrieval of the CIA contribution. All experiments were carried out at room temperature; the maximum pressure of oxygen in the cell was kept around 1 bar for the measurements near the center of the A-band and was as high as 5 bar in the band wings, where the absorption coefficients are small.

The measured extinction coefficients are then corrected for Rayleigh scattering. The contribution of the Rayleigh scattering $(\sigma)$ is given by ${ }^{30}$

$$
\sigma(\bar{\nu})=\frac{24 \bar{\nu}^{4} \pi^{3}\left(n_{s}^{2}-1\right)^{2}}{N_{s}^{2}\left(n_{s}^{2}+2\right)^{2}} \frac{6+3 \rho_{n}}{6-7 \rho_{n}}
$$

where $\bar{\nu}$ is the frequency in wavenumbers, $n_{s}$ is the refraction index, $N_{s}$ is the molecular number density per $\mathrm{cm}^{3}$, and $\rho_{n}$ is the so-called depolarization factor, related to the nonspherical nature of oxygen molecules. The last term of Eq. (8) is known as the King correction factor $(F(\bar{\nu}))$ and is given by ${ }^{31}$

$$
\begin{aligned}
F(\bar{\nu}) \equiv & \frac{6+3 \rho_{n}}{6-7 \rho_{n}}=1.096+1.385 \times 10^{-11}\left(\bar{\nu} / \mathrm{cm}^{-1}\right)^{2} \\
& +1.448 \times 10^{-20}\left(\bar{\nu} / \mathrm{cm}^{-1}\right)^{4},
\end{aligned}
$$

The refraction index is given by

$$
\left(n_{s}-1\right) \times 10^{8}=A+\frac{B}{4.09 \times 10^{9}-\left(\bar{\nu} / \mathrm{cm}^{-1}\right)^{2}} .
$$

The values of the coefficients $A=21351.1$ and $B$ $=21.85670 \times 10^{12}$ for the frequencies below $18315 \mathrm{~cm}^{-1}$ are from Bates. ${ }^{31}$ Using the above equations, the measured extinction of oxygen was corrected for Rayleigh scattering. It should be noted that the relative contribution of scattering depends on the spectral region within the A band: in the central part of the band scattering is negligibly small, while near 12970 and $13280 \mathrm{~cm}^{-1}$ its contribution may be almost $30 \%$ (at 1 amagat).

\section{RESULTS}

As described in Sec. III C, the density dependent extinction coefficients are first corrected for the reflectivity of the mirrors and for the Rayleigh extinction. In the resulting absorption coefficients we distinguish two contributions: the allowed magnetic dipole absorption and absorption by transient $\mathrm{O}_{2}-\mathrm{O}_{2}$ collision pairs. The CIA is strictly quadratic in nature. In the wings of the absorption lines, where the measurements were performed, the LM model predicts a nearly perfect quadratic behavior.

The interpretation of the observed pressure dependence in LM and CIA is of course model dependent, but the observed total quadratic coefficient describing the absorption in 
TABLE I. The averaged measured binary absorption coefficients for various excitation energies in wavenumbers in between the P-lines (P...P...-P...Q...) and the region of the missing Q-branch $(\mathrm{Q})$ of the oxygen A-band, as well as in the region beyond the R-branch bandhead (R).

\begin{tabular}{|c|c|c|c|c|c|}
\hline & $\begin{array}{l}\text { Wavenumber } \\
\left(\mathrm{cm}^{-1}\right)\end{array}$ & $\begin{array}{c}\text { Binary absorption coefficient } \\
\quad\left(10^{-8} \mathrm{~cm}^{-1} \mathrm{amg}^{-2}\right)\end{array}$ & & $\begin{array}{l}\text { Wavenumber } \\
\left(\mathrm{cm}^{-1}\right)\end{array}$ & $\begin{array}{c}\text { Binary absorption coefficien } \\
\quad\left(10^{-8} \mathrm{~cm}^{-1} \mathrm{amg}^{-2}\right)\end{array}$ \\
\hline $\mathrm{P}_{33} \mathrm{P}_{33}-\mathrm{P}_{35} \mathrm{Q}_{34}$ & 12969.0 & $2.7 \pm 0.1$ & $\mathrm{P}_{5} \mathrm{P}_{5}-\mathrm{P}_{7} \mathrm{Q}_{6}$ & 13103.4 & $212 \pm 3$ \\
\hline $\mathrm{P}_{31} \mathrm{P}_{31}-\mathrm{P}_{33} \mathrm{Q}_{32}$ & 12980.6 & $3.7 \pm 0.2$ & $\mathrm{P}_{3} \mathrm{P}_{3}-\mathrm{P}_{5} \mathrm{Q}_{4}$ & 13109.4 & $219 \pm 3$ \\
\hline $\mathrm{P}_{31} \mathrm{P}_{31}-\mathrm{P}_{33} \mathrm{Q}_{32}$ & 12985.2 & $4.0 \pm 0.2$ & $\mathrm{P}_{3} \mathrm{P}_{3}-\mathrm{P}_{5} \mathrm{Q}_{4}$ & 13109.8 & $203 \pm 3$ \\
\hline $\mathrm{P}_{29} \mathrm{P}_{29}-\mathrm{P}_{31} \mathrm{Q}_{30}$ & 12995.0 & $5.25 \pm 0.15$ & $\mathrm{P}_{1} \mathrm{P}_{1}-\mathrm{P}_{3} \mathrm{Q}_{2}$ & 13115.8 & $166 \pm 3$ \\
\hline $\mathrm{P}_{27} \mathrm{P}_{27}-\mathrm{P}_{29} \mathrm{Q}_{28}$ & 13004.2 & $6.4 \pm 0.2$ & $\mathrm{P}_{1} \mathrm{P}_{1}-\mathrm{P}_{3} \mathrm{Q}_{2}$ & 13116.0 & $161 \pm 3$ \\
\hline $\mathrm{P}_{27} \mathrm{P}_{27}-\mathrm{P}_{29} \mathrm{Q}_{28}$ & 13006.2 & $6.4 \pm 0.2$ & Q & 13121.0 & $67 \pm 1$ \\
\hline $\mathrm{P}_{25} \mathrm{P}_{25}-\mathrm{P}_{27} \mathrm{Q}_{26}$ & 13016.4 & $8.1 \pm 0.3$ & $\mathrm{Q}$ & 13122.0 & $60 \pm 1$ \\
\hline $\mathrm{P}_{25} \mathrm{P}_{25}-\mathrm{P}_{27} \mathrm{Q}_{26}$ & 13018.0 & $8.8 \pm 0.3$ & Q & 13123.0 & $61 \pm 1$ \\
\hline $\mathrm{P}_{23} \mathrm{P}_{23}-\mathrm{P}_{25} \mathrm{Q}_{24}$ & 13028.0 & $12.7 \pm 0.4$ & $\mathrm{R}$ & 13170.0 & $23.0 \pm 0.4$ \\
\hline $\mathrm{P}_{21} \mathrm{P}_{21}-\mathrm{P}_{23} \mathrm{Q}_{22}$ & 13037.5 & $18.4 \pm 0.5$ & $\mathrm{R}$ & 13175.0 & $18.2 \pm 0.3$ \\
\hline $\mathrm{P}_{19} \mathrm{P}_{19}-\mathrm{P}_{21} \mathrm{Q}_{20}$ & 13047.0 & $28.6 \pm 0.8$ & $\mathrm{R}$ & 13185.0 & $14.4 \pm 0.2$ \\
\hline $\mathrm{P}_{17} \mathrm{P}_{17}-\mathrm{P}_{19} \mathrm{Q}_{18}$ & 13056.0 & $44 \pm 1$ & $\mathrm{R}$ & 13190.0 & $13.5 \pm 0.2$ \\
\hline $\mathrm{P}_{15} \mathrm{P}_{15}-\mathrm{P}_{17} \mathrm{Q}_{16}$ & 13065.6 & $77.0 \pm 1.5$ & $\mathrm{R}$ & 13195.0 & $12.5 \pm 0.2$ \\
\hline $\mathrm{P}_{13} \mathrm{P}_{13}-\mathrm{P}_{15} \mathrm{Q}_{14}$ & 13072.0 & $105 \pm 2$ & $\mathrm{R}$ & 13200.0 & $12.0 \pm 0.2$ \\
\hline $\mathrm{P}_{13} \mathrm{P}_{13}-\mathrm{P}_{15} \mathrm{Q}_{14}$ & 13073.6 & $100 \pm 2$ & $\mathrm{R}$ & 13210.0 & $11.1 \pm 0.2$ \\
\hline $\mathrm{P}_{11} \mathrm{P}_{11}-\mathrm{P}_{13} \mathrm{Q}_{12}$ & 13080.2 & $146 \pm 2$ & $\mathrm{R}$ & 13220.0 & $10.2 \pm 0.2$ \\
\hline $\mathrm{P}_{11} \mathrm{P}_{11}-\mathrm{P}_{13} \mathrm{Q}_{12}$ & 13081.4 & $130 \pm 2$ & $\mathrm{R}$ & 13230.0 & $9.5 \pm 0.2$ \\
\hline $\mathrm{P}_{9} \mathrm{P}_{9}-\mathrm{P}_{11} \mathrm{Q}_{10}$ & 13089.0 & $162 \pm 3$ & $\mathrm{R}$ & 13240.0 & $8.9 \pm 0.2$ \\
\hline $\mathrm{P}_{9} \mathrm{P}_{9}-\mathrm{P}_{11} \mathrm{Q}_{10}$ & 13089.2 & $167 \pm 3$ & $\mathrm{R}$ & 13250.0 & $8.3 \pm 0.2$ \\
\hline $\mathrm{P}_{7} \mathrm{P}_{7}-\mathrm{P}_{9} \mathrm{Q}_{8}$ & 13096.2 & $192 \pm 3$ & $\mathrm{R}$ & 13260.0 & $7.7 \pm 0.2$ \\
\hline $\mathrm{P}_{7} \mathrm{P}_{7}-\mathrm{P}_{9} \mathrm{Q}_{8}$ & 13096.6 & $202 \pm 3$ & $\mathrm{R}$ & 13270.0 & $7.2 \pm 0.2$ \\
\hline $\mathrm{P}_{5} \mathrm{P}_{5}-\mathrm{P}_{7} \mathrm{Q}_{6}$ & 13103.0 & $211 \pm 3$ & $\mathrm{R}$ & 13280.0 & $6.7 \pm 0.2$ \\
\hline
\end{tabular}

the minima between the P-lines and in the region of the missing Q-branch is a relevant reference information for generating reference spectra. In Table I, the absorption coefficients are given in $\mathrm{cm}^{-1} \mathrm{amg}^{-2}$. The estimated relative error is better than $1.5 \%$ in the middle of the A-band and better than $3 \%$ near the wings of the A-band.

Using the ABC-model we estimated the values of the absorption coefficient in the A-band for frequencies around the minima between the rotational lines and behind the bandhead. As will be described below, the only free remaining parameter of the ABC-model, the interbranch coupling, was set at 0.8 , in order to have a smooth CIA spectrum. This value is not much different from the value of 0.59 derived by Makarov et al. ${ }^{32}$ while studying the millimeter absorption spectrum of oxygen. The ABC model calculations showed that for the spectral points chosen between the lines, the absorption coefficient increased proportional to the square of oxygen density within $0.5 \%$.

\section{A. Determination of the CIA spectrum}

Figure 3 shows a representative experimental ramp of extinction against density at $13123.32 \mathrm{~cm}^{-1}$. In panel (a), each point is the result of an exponential fit of a light decay curve, corrected for the reflectivity of the mirrors. The Rayleigh scattering is shown as the straight line. In panel (b), the observations are corrected for this scattering. The solid line shows the result of density dependent calculations of the ABC model. In panel (c), we have subtracted the calculated model absorption. A small correction on the absorption coefficients is applied at those frequencies where the model ab- sorption coefficients change significantly over the laser bandwidth. This was only necessary for a small number of points close to the resonance frequencies and was achieved by convoluting the calculated model absorption with the Gaussian

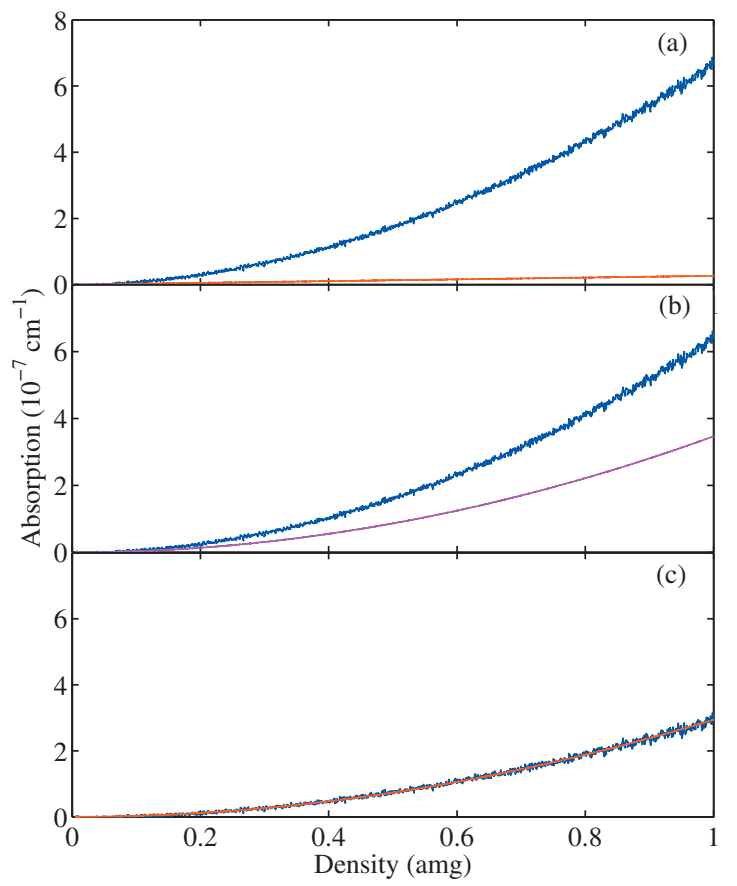

FIG. 3. Sketch of the procedure. The data are taken at $13123.32 \mathrm{~cm}^{-1}$. (a). Measured extinction (blue) and Rayleigh scattering (red). (b). Measured absorption (blue), resulting from the subtraction of the Rayleigh scattering from the extinction, and calculated absorption (purple), obtained using the ABC-model. (c). The CIA (blue) resulting from subtraction of the modeled absorption from the measured absorption; the CIA is fitted with a quadratic function (red). 


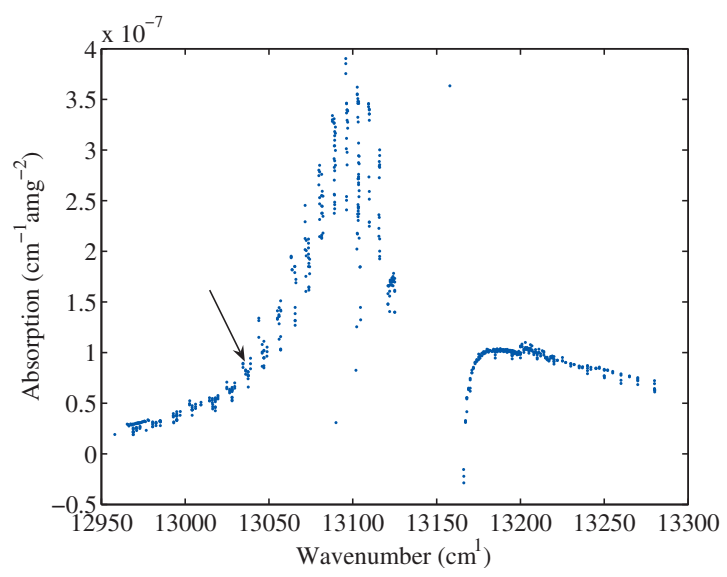

FIG. 4. Retrieved CIA coefficients assuming a sum of Voigts realization of the A-band spectrum without LM. Note the apparent negative values for coefficients beyond the bandhead at $13167 \mathrm{~cm}^{-1}$. No reliable data points could be taken below the R-branch bandhead. The arrow points toward a minimum where a nonsmooth structure can be observed.

laser profile. The resulting absorption density $(D)$ ramp is attributed to CIA and is fitted with a one parameter quadratic fit, i.e.,

$$
a(D)=D^{2} b
$$

The constant $b$ is the collision induced absorption coefficient $\left(\mathrm{cm}^{-1} \mathrm{amg}^{-2}\right)$. We required from the behavior of the CIA that the fitted curve through the measured CIA is quadratic over the full ramp.

\section{Voigt lines}

In order to check whether we needed to take LM into account to derive a realistic CIA spectrum, we performed the analysis described above not using the $\mathrm{ABC}$ model but a sum of Voigt line shapes based on HITRAN08 reference data of the A-band. The resulting apparent CIA spectrum is displayed in Fig. 4.

The absorption coefficients are given at a reference density of 1 amagat. The values can be interpreted as the quadratic absorption coefficients in $\mathrm{cm}^{-1}$ amagat ${ }^{-2}$. All data points of Fig. 4 are the result of independent pressure ramps. The resulting CIA spectrum is rather smooth, especially beyond the bandhead in the R-branch. We note that we have no data-points for frequencies in the R-branch itself. The higher line density of the branch results in high absorption coefficients due to the allowed transitions with the consequence that the contribution of CIA is too small. Here, the CIA contributes less then $0.5 \%$ at 1 amagat to the total absorption. The absence of direct measurements makes it difficult to estimate the band-integrated CIA coefficient. Figure 4 compiles all data points that were taken over a long period of time, even including a move of the whole instrument.

The spread of the points within one minimum is not purely statistical. This can be observed, for example, from the observed CIA coefficients around $13040 \mathrm{~cm}^{-1}$. Here the coefficients are smaller at the minima than closer to the lines. Due to the nature of CIA, we implicitly assume the spectrum to be without such high frequency structure. Also, the apparent CIA in the region of the Q-branch is small in the view of

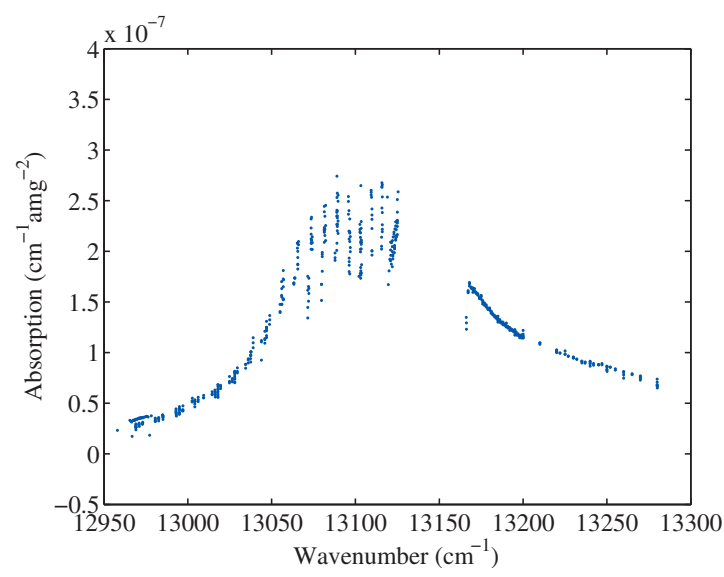

FIG. 5. Retrieved CIA coefficients in $\mathrm{cm}^{-1}$ amagat ${ }^{-2}$, assuming the adjustable branch coupling model, of the A-band region. The branch coupling constant has been set to 0.8 resulting in a smooth CIA spectrum.

the fact that the CIA spectrum under the P- and R-branch will be of similar magnitude and that sharp structures are absent. The large spread near the maximum of the P-branch is partially due to the small contribution of CIA and partially due to the incorrectness of the sum of Voigts spectrum. The most convincing argument for the need of introducing LM are the nonphysical negative values of the CIA coefficients that are derived for frequencies beyond the bandhead.

\section{Voigt lines including line mixing}

Subsequently, we have made use of the ABC model with one free parameter: the value of the interbranch coupling, $r$. The effects of this value are most obvious in the missing Q-branch region and, to a lesser extent, just beyond the bandhead. The numerical value for $r(0.8)$ was chosen such that the resulting CIA spectrum is smooth. The retrieved CIA coefficients are shown in Fig. 5. Line mixing affects the magnetic dipole absorption by slightly deforming the Voigt line shapes and shifting intensity from the edges of the band to its center, resulting in a change of the CIA spectrum. The negative values and the local minima in the CIA spectrum in between the P-lines with high rotational quantum numbers have disappeared. Also the maximum intensity is reduced. However, we still find that the retrieved CIA coefficients within a number of individual minima are not smooth but show relative large variations that are not random. For frequencies where $N>9$, the slope is tilted up. The reverse characterizes the observations for $N<9$. In the region just beyond the bandhead, still a few points are observed with a surprising behavior, which has to reflect a remaining systematic model error. We have carefully checked that the wavelength meter did not introduce a small systematic shift in wavelength, which could explain the behavior in each minimum. For the areas below $13075 \mathrm{~cm}^{-1}$ and above $13200 \mathrm{~cm}^{-1}$, the averaged retrieved CIA does not change by introducing LM, due to the large contribution of CIA compared to the model absorption. We stress that we consider the results in Fig. 5 being a smooth retrieved CIA spectrum.

In Fig. 6, we present the CIA spectrum, obtained by taking one averaged value of the CIA coefficient for each minimum given, based on all data close to the minimum. It 


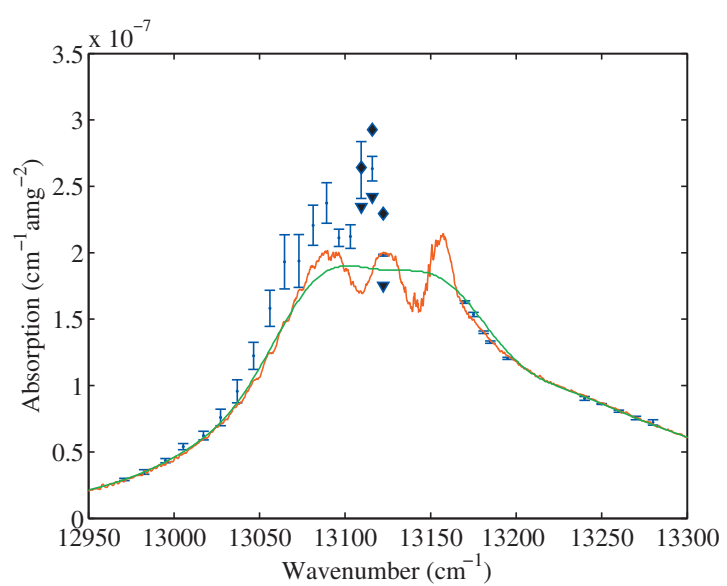

FIG. 6. Averaged CIA coefficients in $\mathrm{cm}^{-1}$ amagat ${ }^{-2}$, assuming the adjustable branch coupling model, of the A-band region. The branch coupling constant has been set to 0.8 resulting in a smooth CIA spectrum. The points are the result of the averaging of multiple points taken in and near the minima between the lines. Also displayed are the results for setting $r$ at 0.7 (diamonds) and 0.9 (triangles). The green line displays the smoothed CIA spectrum obtained by Tran et al. (Ref. 14), while the red line displays their unsmoothed data.

should be realized that each point is the result of many independent measured points, which each are the result of analyzing a few thousand decay curves during a full pressure scan. As was discussed above, part of the errors bar shown in the data-points still have a systematic origin, which reflects that the LM model is not perfect yet. Another explanation for part of the systematic error is the presence of inaccuracies in the HITRAN database. We found that the improved line positions of the isotopologues in HITRAN $08^{20}$ improved the fits with respect to HITRAN04. ${ }^{19}$ The largest part of the error at low rotational lines in the $\mathrm{P}$ branch is caused by the high absorption coefficient at the minima between the lower $\mathrm{P}$ lines due to the allowed magnetic dipole transitions, rendering relatively small CIA contributions. Figure 6 also contains a direct comparison with the data of Tran et al. ${ }^{14}$

As mentioned above, for several minima in between the P-branch we performed detailed measurements of the absorption coefficients taking many points in each minimum. The idea was to use the shape in between the resonances, to optimize the LM model. Also, the need to incorporate LM came from the detailed observations between different lines. The choice of the parameter $r$ is fully based on the overall shape of the CIA spectrum. The detailed shape in between the resonances could not be used for refinement of our LM model. Figure 7 shows the behavior at three positions of the spectrum, displaying how accurate the sum of our LM model and a smooth CIA spectrum based on the results of Fig. 6 describes the observed data. In the upper panel (a) the magnitude of the CIA is only $2 \times 10^{-7} \mathrm{~cm}^{-1}$ amagat $^{-2}$. The isotopologues are visible. The observed deviations at the edges and around the isotopologues result from the rapidly changing absorption, which causes the light from the cavity to decay in a multiexponential fashion. A correction is performed, but the correction method leaves a systematic error underestimating the true absorption. The middle panel (b) shows opposite systematic errors occurring in the missing Q-branch region due to predictions of the LM model. On the
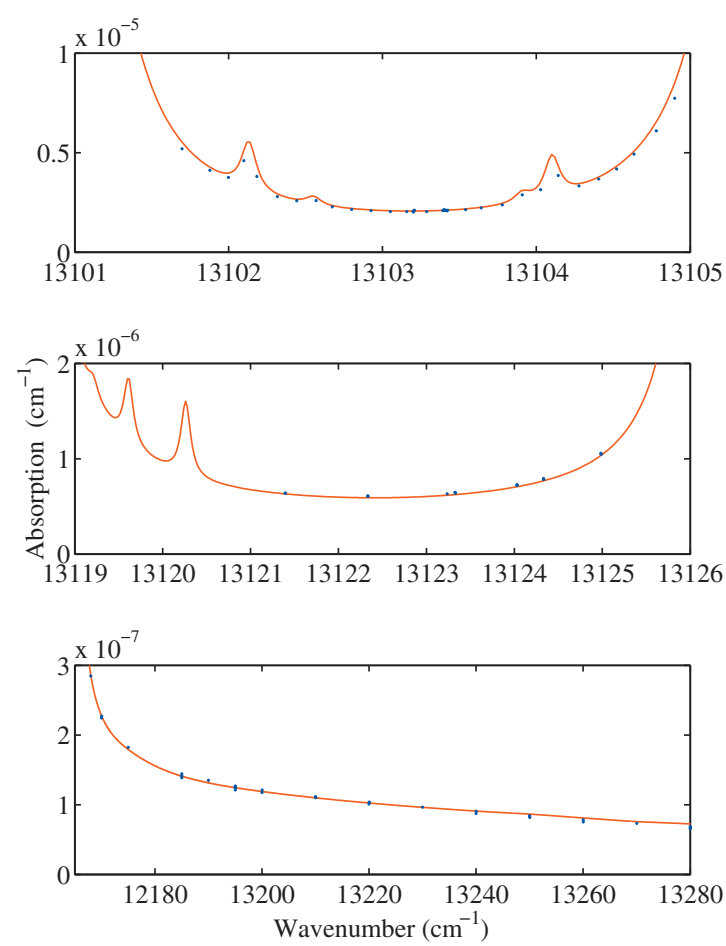

FIG. 7. Detailed measured absorption coefficients (points) at 1 amagat, corrected for Rayleigh scattering, compared to absorption coefficients calculated as the sum of the ABC model with branch coupling constant $r$ is 0.8 and CIA (line). (a). Minimum between lines P7Q6 and P6Q6 in the P-branch. (b). Region of the missing Q branch. (c). Region beyond the bandhead in the R-branch.

right hand side, the measurements are slightly above the model values, while on the left hand side the observations match the model. In the last panel (c), the region above the bandhead is shown. Here, the observations are dominated by the CIA contribution. All data points, resulting from independent pressure scans, form directly a smooth spectrum, which is indicative for the accuracy of the present experiment.

As shown above an important determination of the CIA of oxygen in the A-band region was achieved by Tran et al., ${ }^{14}$ who used FT spectroscopy in combination with pressures up to 150 bar to increase the relative contribution of CIA to the spectrum. It should be noted that the combinations of their LM model and their observations resulted in a CIA spectrum with some structure. Their recommended CIA spectrum has been smoothed in accordance with the expectation that the CIA spectrum should have little structure. Also Tran et al. suggested the possibility of residual errors in their line mixing model. We find that our CIA values agree within $5 \%$ with their data up to $13166 \mathrm{~cm}^{-1}$. The unsmoothed data of Tran et al. (Fig. 6 in Ref. 14) has a structure at $13110 \mathrm{~cm}^{-1}$ which we also find in our data. Although the overall shape of our CIA spectrum is very similar and structure in our data seems to follow their structure, our maximum is significantly larger than that of Tran et al. ${ }^{14}$ Fundamentally, their procedure and our procedure are highly similar. They used spectra based on the sum of Lorentzians combined with a line mixing model based on the energy corrected sudden approximation, which weighs the inelastic cross section as function of angular momentum and angular momentum changes. We used Voigt type line shapes com- 


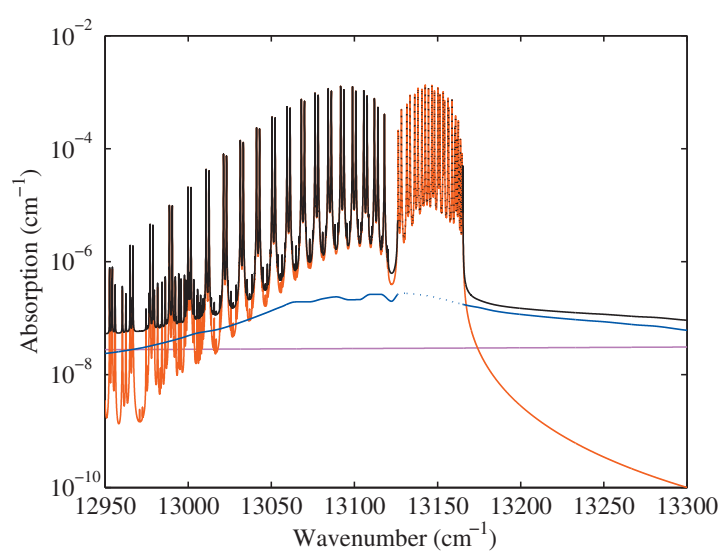

FIG. 8. Summary of the different processes contributing to the extinction by oxygen in region in the A-band at a density of 1 amagat: Rayleigh scattering (purple), prediction of the LM model (red), CIA spectrum as determined in this work (blue), and the sum of all processes are shown (black). The dashes are the region of the R-branch.

bined with the hard collisions assumption, where no memory of the initial state before the collision is used, to describe line mixing. The use of Lorentz lines instead of Voigts agrees with the high pressures employed in Ref. 14. As both our LM models produce the same structure, either the CIA is structured or the LM model needs improvement elsewhere than in effects of the collisions, for example, in HITRAN. ${ }^{20}$ The most likely conclusion is that LM is not accurately described in both models.

Tran et al. ${ }^{14}$ not only published the CIA spectrum of the $\mathrm{O}_{2}-\mathrm{O}_{2}$ collisions but also of the $\mathrm{O}_{2}-\mathrm{N}_{2}$ collisions. The latter is so much narrower than the $\mathrm{O}_{2}-\mathrm{O}_{2}$ spectrum that an estimate of the width of the CIA spectrum based on the available kinetic energy certainly is not possible. In recent experiments in our set-up on the CIA spectrum due to $\mathrm{O}_{2}-\mathrm{N}_{2}$ collisions we corroborate the findings of Tran et al. ${ }^{14}$ and find a rather narrow spectrum even with some structure. ${ }^{33}$ The striking difference between the CIA related to $\mathrm{O}_{2}-\mathrm{O}_{2}$ collisions in comparison to that for $\mathrm{O}_{2}-\mathrm{N}_{2}$ collisions has been noticed before and cannot yet be accounted for by the models based on quadrupole interaction induced dipole transitions as formulated by Tipping and co-workers. ${ }^{24,25}$

It is clear that we have been able to determine the relative contributions of Rayleigh scattering, the allowed magnetic dipole transition and the CIA in oxygen. The resulting total spectrum and the relative contributions of the different processes are displayed in Fig. 8. The data are plotted on a logarithmic scale, to show the influence of the various processes on the spectrum.

At 1 amagat, Rayleigh scattering dominates in the minima between P-lines with large rotational quantum numbers (below $12985 \mathrm{~cm}^{-1}$ ). CIA dominates in the region beyond the bandhead in the R-branch $\left(>13170 \mathrm{~cm}^{-1}\right)$ as well as in a small region at the minima in the P-branch (from 12985 to $13037 \mathrm{~cm}^{-1}$ ). In the remaining region, especially at the frequencies of the R-branch (from 13130 to $13170 \mathrm{~cm}^{-1}$ ), the spectrum is dominated by the magnetic dipole absorption of oxygen. At lower densities, Rayleigh scattering will dominate, due to its linear dependence on density, whereas the absorption in the minima and the CIA are quadratic in their density dependence.

The shape and width of CIA spectra can be understood from some general principles. In principle absorption takes place by short lived collision complexes, implying that the spectrum cannot display sharp features. During the collision internal energy and kinetic energy can be exchanged, hence part of the broadening will be determined by the kinetic energy distribution of the collision partners. Most interestingly, molecular properties as the interaction potential, the magnitude of the transient dipole moment being formed, and the effects of having two triplet-state collision partners contribute to the detailed shape of the spectrum. We note that in the fundamental band of molecular oxygen, the CIA spectrum does show structure, reminiscent of rotational structure. ${ }^{34} \mathrm{~A}$ later paper showed, by using theoretical calculations, that the structure is due to metastable dimers. ${ }^{35}$ However, the equilibrium density of dimers is expected to be quadratic in density. Hence we cannot distinguish them on the basis of the density dependence and the dimers contribute to the CIA spectrum and may add a weak, sharp structure to a CIA spectrum.

\section{CONCLUSIONS}

The renewed interest in CIA and LM in the case of systems such as oxygen stems partially from the ever increasing level of details needed to characterize the Earth' atmosphere. The need for understanding of global warming has been translated in the necessity of assessing $\mathrm{CO}_{2}$ concentrations to within $1 \%$. With such an accuracy not only the increase in $\mathrm{CO}_{2}$ can be monitored but even localized emissions may become measurable by remote sensing. Retrieval from satellite data always requires the knowledge of the air mass factor and effects of aerosol scattering. Quantitative determination of the amount of molecular oxygen can be used to determine these effects. Using CRDS we have been able to determine with very high accuracy the absolute extinction coefficients of molecular oxygen at atmospheric pressures, especially in between the resonance lines. These positions in the spectrum are highly relevant as variations in absorption as function of observation geometry and air mass are mainly due to these line wings as the line centers are optically very thick. In this work we have used the density dependence of extinction to identify LM and CIA contribution. Accurate determinations have been possible. It has been impossible to obtain information on the CIA in the frequency region of the R-branch at our pressures. The accuracy of the CIA spectrum would be larger when a LM model can be used that is validated against large sets of data, as we believe that LM model still is a source of systematic errors. Predoi-Cross et al. ${ }^{8}$ also retrieved LM parameters by assessing at low pressures the full line shape of the allowed transitions. This method forms an alternative way to distinguish between different LM models. The results clearly indicate that a need exists for assessing the CIA spectrum and full line shapes starting from first principles. 


\section{ACKNOWLEDGMENTS}

We thank Professor Dr. Tonkov of the St. Petersburg University for his inspiration. We thank Dr. Tran and Dr. Hartmann for providing us with numerical data of their CIA spectra of pure $\mathrm{O}_{2}$. We thank Professor Dr. Predoi-Cross for helping us with an attempt to derive the CIA using Galatry type line shapes. We acknowledge the financial support from NWO (Echo project).

${ }^{1}$ B. van Diedenhoven, O. P. Hasekamp, and I. Aben, Atmos. Chem. Phys. 5, 2109 (2005).

${ }^{2}$ Z. M. Kuang, J. Margolis, G. Toon, D. Crisp, and Y. Yung, Geophys. Res. Lett. 29, 1716 (2002).

${ }^{3}$ R. H. Dicke, Phys. Rev. 89, 472 (1953).

${ }^{4}$ U. Fano, Phys. Rev. 131, 259 (1963).

${ }^{5}$ L. Frommhold, Collision-induced Absorption in Gases, Cambridge Monographs on Atomic, Molecular, and Chemical Physics Vol. 2, 1st ed. (Cambridge University Press, Cambridge, 1993).

${ }^{6}$ L. R. Brown and C. Plymate, J. Mol. Spectrosc. 199, 166 (2000).

${ }^{7}$ D. J. Robichaud, J. T. Hodges, P. Maslowski, L. Y. Yeung, M. Okumura, C. E. Miller, and L. R. Brown, J. Mol. Spectrosc. 251, 27 (2008).

${ }^{8}$ A. Predoi-Cross, K. Harnbrook, R. Keller, C. Povey, I. Schofield, D. Hurtmans, H. Over, and G. Ch. Mellau, J. Mol. Spectrosc. 248, 85 (2008).

${ }^{9}$ L. Galatry, Phys. Rev. 122, 1218 (1961).

${ }^{10}$ S. G. Rautian and I. I. Sobelman, Soviet Physics Uspekhi-USSR 9, 701 (1967).

${ }^{11}$ G. C. Tabisz, E. J. Allin, and H. L. Welsh, Can. J. Phys. 47, 2859 (1969).

${ }^{12}$ Although the amagat is not an SI unit, we prefer it as unit of relative density. One amagat is defined as amg $=n / n_{L}$, where $n$ is the molecular density per $\mathrm{cm}^{3}$ and $n_{L}$ is the Loschmidt number $\left(2.686763 \times 10^{19} \mathrm{~mol}-\right.$ ecules $\mathrm{cm}^{-3}$, the number of molecules at $1 \mathrm{~atm}$ of pressure and $273 \mathrm{~K}$ ).

${ }^{13}$ G. D. Greenblatt, J. J. Orlando, J. B. Burkholder, and A. R. Ravishankara, J. Geophys. Res., [Atmos.] 95, 18577 (1990).

${ }^{14}$ H. Tran, C. Boulet, and J. M. Hartmann, J. Geophys. Res., [Atmos.] 111, 15210 (2006).

${ }^{15}$ M. V. Tonkov, N. N. Filippov, Y. M. Timofeyev, and A. V. Polyakov, J. Quant. Spectrosc. Radiat. Transf. 56, 783 (1996).

${ }^{16}$ N. N. Filippov and M. V. Tonkov, Spectrochim. Acta, Part A 52, 901 (1996).

${ }^{17}$ H. D. Babcock and L. Herzberg, Astrophys. J. 108, 167 (1948).

${ }^{18}$ S. G. Rautian, Opt. Spectrosc. 102, 315 (2007).

${ }^{19}$ L. S. Rothman, D. Jacquemart, A. Barbe, D. C. Benner, M. Birk, L. R. Brown, M. R. Carleer, C. Chackerian, K. Chance, L. H. Coudert, V.
Dana, V. M. Devi, J. M. Flaud, R. R. Gamache, A. Goldman, J. M. Hartmann, K. W. Jucks, A. G. Maki, J. Y. Mandin, S. T. Massie, J. Orphal, A. Perrin, C. P. Rinsland, M. A. H. Smith, J. Tennyson, R. N. Tolchenov, R. A. Toth, J. Vander Auwera, P. Varanasi, and G. Wagner, J. Quant. Spectrosc. Radiat. Transf. 96, 139 (2005).

${ }^{20}$ L. S. Rothman, I. E. Gordon, A. Barbe, D. Chris Benner, P. E. Bernath, M. Birk, V. Boudon, L. R. Brown, A. Campargue, J. P. Champion, K. Chance, L. H. Coudert, V. Dana, V. M. Devi, S. Fally, J. M. Flaud, R. R. Gamache, A. Goldman, D. Jacquemart, I. Kleiner, N. Lacome, W. J. Lafferty, J. Y. Mandin, S. T. Massie, S. N. Mikhailenko, C. E. Miller, N. Moazzen-Ahmadi, O. V. Naumenko, A. V. Nikitin, J. Orphal, V. I. Perevalov, A. Perrin, A. Predoi-Cross, C. P. Rinsland, M. Rotger, M. Simeckova, M. A. H. Smith, K. Sung, S. A. Tashkun, J. Tennyson, R. A. Toth, A. C. Vandaele, and J. Vander Auwera, J. Quant. Spectrosc. Radiat. Transf. 110, 533 (2009).

${ }^{21}$ M. O. Bulanin, A. B. Dokuchaev, M. V. Tonkov, and N. N. Filippov, J. Quant. Spectrosc. Radiat. Transf. 31, 521 (1984).

${ }^{22}$ I. M. Grigoriev, R. Le Doucen, A. Benidar, N. N. Filippov, and M. V. Tonkov, J. Quant. Spectrosc. Radiat. Transf. 58, 287 (1997).

${ }^{23}$ A. V. Polyakov, Y. M. Timofeev, M. V. Tonkov, and N. N. Filippov, Izv., Acad. Sci., USSR, Atmos. Oceanic Phys. 34, 328 (1998).

${ }^{24}$ R.H. Tipping, Alex Brown, Q. Ma, and C. Boulet, J. Mol. Spectrosc. 209, 88 (2001).

${ }^{25}$ R. H. Tipping, Q. Ma, C. Boulet, and J.-M. Hartmann, J. Mol. Struct. 742, 83 (2005) (Molecular Spectroscopy and Structure-A Collection of Invited Papers in Honor of Dr. Walter J. Lafferty.).

${ }^{26}$ A. O'Keefe and D. A. G. Deacon, Rev. Sci. Instrum. 59, 2544 (1988).

${ }^{27}$ G. Berden and R. Engeln, Cavity Ring-Down Spectroscopy: Techniques and Applications (Wiley, New York, 2009).

${ }^{28}$ G. Berden, R. Peeters, and G. Meijer, Int. Rev. Phys. Chem. 19, 565 (2000).

${ }^{29}$ H. Naus and W. Ubachs, Opt. Lett. 25, 347 (2000).

${ }^{30}$ E. J. McCartney, Optics of the Atmosphere, Scattering by Molecules and Particles, 1st ed. (Wiley, New York, 1976).

${ }^{31}$ D. R. Bates, Planet. Space Sci. 32, 785 (1984).

${ }^{32}$ D. S. Makarov, N. N. Filippov, and M. Yu. Tretyakov, Opt. Spectrosc. 105, 7 (2008)

${ }^{33}$ Frans Reinier Spiering, Maria Kiseleva, Nikolai N. Filippov, Hans Naus, Bas Lieshout, and Wim J. van der Zande, "Absolute determination of the effects of collisions on oxygen absorption in the a-band using cavity ring-down spectroscopy: Interaction with $\mathrm{N}_{2}$ " (unpublished).

${ }^{34}$ G. Moreau, J. Boissoles, C. Boulet, R. H. Tipping, and Q. Ma, J. Quant. Spectrosc. Radiat. Transf. 64, 87 (2000).

${ }^{35}$ G. Moreau, J. Boissoles, R. Le Doucen, C. Boulet, R. H. Tipping, and Q. Ma, J. Quant. Spectrosc. Radiat. Transf. 70, 99 (2001). 